\title{
Ejercicios físicos en los niveles de Síndrome de Burnout y la Variabilidad de la Frecuencia Cardíaca de estudiantes universitarios: un estudio de intervención
}

Yury Rosales-Ricardo ${ }^{1,4}{ }^{(\mathbb{D})}$, Marco Vinicio Caiza-Ruiz ${ }^{2}{ }^{(1)}$, Mauricio Sánchez-Cañizares ${ }^{3}{ }^{(D)}$, José Pedro Ferreira ${ }^{4}$ (D)

\begin{abstract}
RESUMEN
Los programas de intervención para prevenir o tratar el Síndrome de Burnout son esenciales para mejorar la salud de los estudiantes. Pero la mayoría de las investigaciones realizadas son intervenciones psicológicas y cognitivas.

Objetivo: Determinar los efectos de los ejercicios físicos en los niveles de Síndrome de Burnout y la Variabilidad de la Frecuencia Cardíaca en estudiantes universitarios.

Métodos: Estudio experimental con pre-test y post-test, con 2 grupos de intervención y un grupo de control. La muestra fue probabilística y estratificada con participación proporcional. Instrumentos utilizados: Maslach Burnout Inventory Students Survey (MBI-SS) y Variabilidad de la Frecuencia Cardíaca (HRV): cálculo de la media RR, SDNN y RMSSD. Para el ejercicio físico, se aplicaron ejercicios aeróbicos y de fuerza durante 3 sesiones semanales de una hora, en días alternos, durante 16 semanas.

Resultados: El grupo de aeróbicos con Agotamiento MBI-SS fue mayor, reduciendo sus niveles en un $26,4 \%(d=0,532)$, en Cinismo $(-21,06, d=0,252)$ y en Eficacia $(-13,11,0,397)$. Grupo de fuerza en Cinismo $(-27,38, d=0,315)$, en Eficacia $(-21,69, d=0,704)$, Agotamiento $(-19,55, d=0,299)$. El grupo de control Agotamiento aumentó en un $10,26 \%(d=0,128)$. En la HVR, con el grupo aeróbico el SDNN tuvo el mayor cambio porcentual, con un aumento del $24,82 \%$, sobre la media RR y RMSSD (14,40 \% y $16,45 \%)$. En el grupo de fuerza y en el grupo de control $(21,77 \%, 14,24 \%, 12,60 \%$; y $12,59 \%$, $4,97 \%$ y $4,99 \%$ respectivamente). Hubo un cambio en el RR medio en los grupos aeróbico y de fuerza $(\mathrm{d}=1,281$ y 1,328$)$. Conclusiones: Efecto de reducción del síndrome de burnout y efecto contrario de incremento de valores del HRV.
\end{abstract}

Palabras clave: Ejercicios físicos, Síndrome de burnout, Frecuencia cardíaca, Salud mental.

1. University San Gregorio of Portoviejo. Medical School. Ecuador

2. National University of Chimborazo. Faculty of Health Sciences. Career in Physical and Sports Therapy. Ecuador

3. University of Ambato. Faculty of Human Science and Education. Ecuador

4. University of Coímbra. Faculty of Sports Sciences and Physical Education. Portugal 


\section{INTRODUCCIÓN}

El Síndrome de Burnout (SB) es un problema de salud de gran repercusión social hoy en día. En un inicio el SB se estudiaba exclusivamente en profesionales de la salud, pero en la actualidad estos estudios se han iniciado en otras poblaciones de profesionales e incluso en estudiantes universitarios ${ }^{1}$.

En la clasificación internacional de enfermedades se encuentra este síndrome: Factores que influyen en el estado de salud y el contacto con los servicios de salud. Dentro de la Z 73: Problemas relacionados con las dificultades para afrontar la vida. Y específicamente, en el Z 73.0: Síndrome de burnout ${ }^{2}$. Freudenberger ${ }^{3}$ describe el SB como una sensación de fracaso y una existencia agotada o desgastada como resultado de una sobrecarga de energía, recursos personales o fuerza espiritual del trabajador. Por su parte, Cherniss ${ }^{4}$ es uno de los primeros autores en destacar la importancia del trabajo como precursor de la aparición del SB y lo define como "los cambios personales negativos que se producen con el tiempo en los trabajadores con trabajos frustrantes o demandas excesivas".

Maslach y Jackson ${ }^{5}$ entienden el SB como "un síndrome tridimensional caracterizado por el agotamiento emocional, la despersonalización y la reducción de la autorrealización". Esta definición, que no se aparta del supuesto de que las variables del trabajo son los determinantes últimos de la aparición del agotamiento, tiene la importancia de no ser teórica, sino que es una consecuencia empírica del estudio que los autores desarrollaron.

Actualmente, la mayoría de los estudios se enmarcan dentro del enfoque tridimensional que el síndrome se compone de agotamiento emocional, despersonalización y pérdida de la autorrealización en el lugar de trabajo ${ }^{6-11}$ y en el caso de los estudiantes las tres dimensiones son: agotamiento emocional, cinismo y eficacia académica ${ }^{12-14}$. Los estudiantes de educación superior, como los profesionales, se enfrentan a las presiones y sobrecargas del trabajo académico. Hasta la fecha, se han realizado muy pocos estudios de intervención con ejercicio físico en estudiantes universitarios con SB y qué efectos tendrían estos en la variabilidad de la frecuencia cardiaca y en el propio síndrome.

Los programas de intervención para prevenir o tratar el SB son esenciales para mejorar la salud de los trabajadores y los estudiantes. Los estudios de revisión ${ }^{15,16}$ han demostrado que la gran mayoría de las investigaciones realizadas y encontradas son intervenciones psicológicas y cognitivas. Las personas que perciben mayores niveles de salud son los que presentan mayor Variabilidad de la Frecuencia Cardiaca (HRV). Una mayor activación vagal en reposo se relaciona con una mayor calidad de vida en relación con la salud. El análisis de la HRV es un buen marcador del estado de salud y puede ayudar a diagnosticar rápidamente y con facilidad estados de estrés, burnout, fatiga, sobreentrenamiento, agotamiento o ansiedad, en la población general ${ }^{17}$. La HVR es un excelente biomarcador cardiovascular para la prevención y detección temprana del SB ${ }^{18}$.

Este estudio aborda el SB en estudiantes universitarios y propone la práctica de ejercicio físico como intervención. El tema abordado en el estudio se considera que es relevante e importante, ya que los estudiantes universitarios experimentan presión para completar las actividades, estrés, horarios exhaustivos y sobrecarga de actividades, que posteriormente contribuyen al desarrollo del SB. Además, la intervención propuesta es coherente con estudios anteriores en la literatura, en los que la práctica de ejercicio físico contribuye al alivio de la ansiedad, el estrés y a una mejor calidad de vida $19-21$. Si la intervención resulta eficaz, esto sugeriría que existe una estrategia sencilla, rentable y accesible para reducir el SB en esta importante y amplia población académica. Los resultados de esto podrían utilizarse para proporcionar una mejor política y práctica basada en la evidencia a los estudiantes universitarios de diferentes carreras y a los responsables de la política sanitaria en relación con el efecto del ejercicio en el bienestar de los estudiantes universitarios.

Por tanto, el principal objetivo de este estudio fue determinar los efectos de los ejercicios físicos en los niveles de Síndrome de Burnout y la Variabilidad de la Frecuencia Cardíaca en estudiantes universitarios.

\section{MATERIAL Y MÉTODOS}

\section{Diseño del estudio}

Se realizó un estudio experimental con pretest y postest, con dos grupos de intervención (grupo de ejercicios aeróbicos y grupo de ejercicios de fuerzas) y un grupo de control (sin ejercicios). Los participantes de cada grupo se distribuyeron aleatoriamente, utilizando un muestreo aleatorio simple. 


\section{Participantes}

La muestra partió de la población de estudiantes diagnosticados con el síndrome de burnout en la Universidad de Ambato en Ecuador, donde se estudió una amplia variedad de carreras universitarias (en ciencias de la salud, ingeniería, ciencias de la educación, economía y negocios, agronomía, derecho, turismo y ciencias sociales). El tamaño de la muestra se obtuvo a partir del error estándar de la distribución de la muestra proporcional y del valor crítico $\mathrm{K}$, correspondiente al nivel de confianza elegido (ver figura 1).
La muestra total fue de 81 participantes que fueron seleccionados al azar. No hubo diferencias entre las condiciones al principio del estudio y se dividieron en tres grupos de composición similar, utilizando un muestreo aleatorio simple. El grupo de ejercicio aeróbico estuvo formado por 28 estudiantes, todos recibieron la intervención, aunque para el análisis estadístico sólo se tuvieron en cuenta 26, ya que 2 no realizaron una práctica regular de al menos 3 días por semana. El grupo de ejercicios de fuerza estaba formado por 26 estudiantes, todos ellos recibieron también la intervención regular, aunque para el análisis estadístico sólo se tuvieron

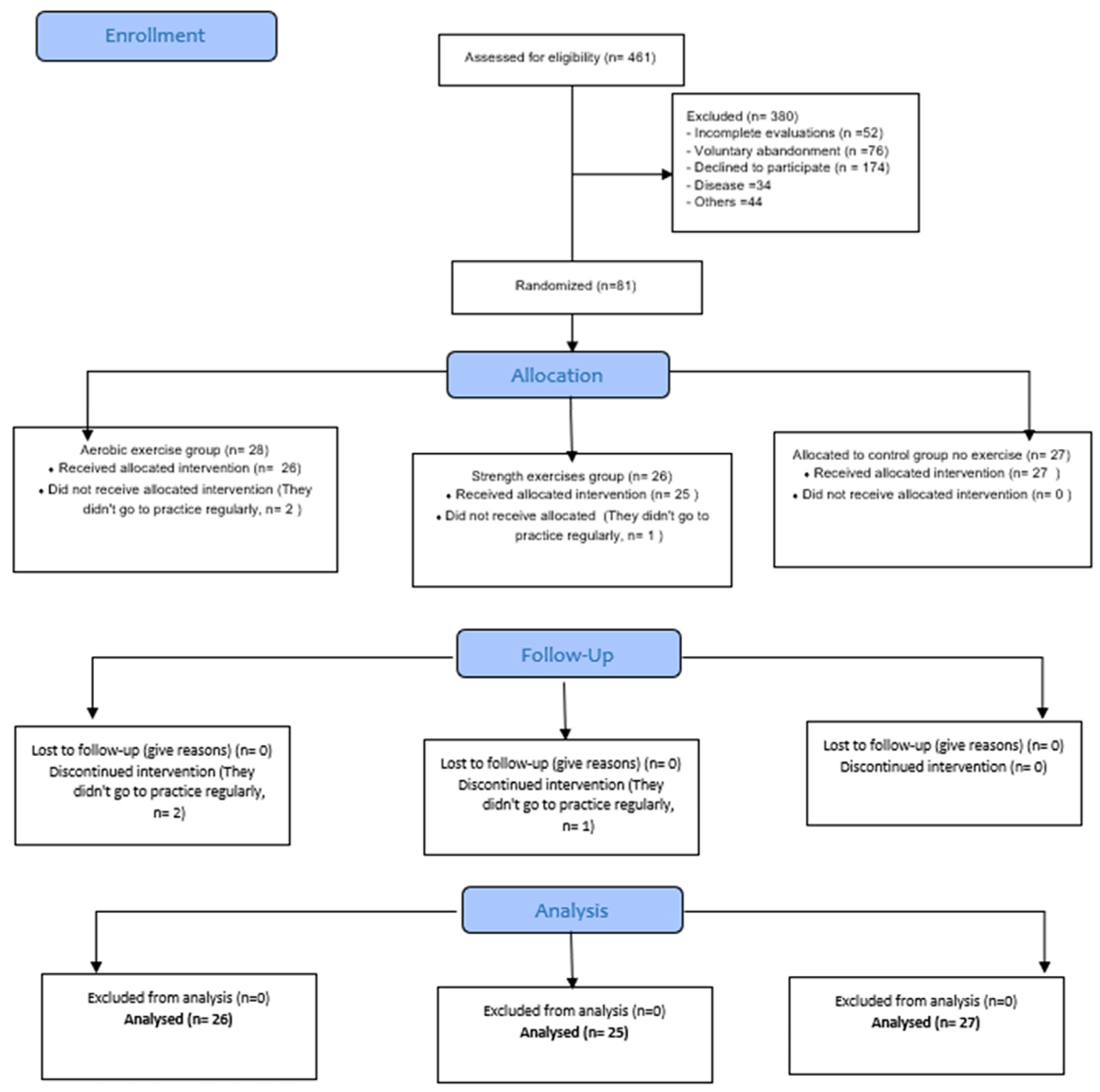

Figura 1. Diagrama de flujo del protocolo de estudio. 
en cuenta 25, ya que uno no realizaba las prácticas regularmente. El grupo de control, no recibió ninguna intervención, pero fue objeto de seguimiento, estuvo integrado por 27 estudiantes.

\section{Instrumentos}

- Síndrome de Burnout en estudiantes: se utilizó el Maslach Burnout Inventory Students Survey (MBI-SS) ${ }^{22}$. Está estructurado en 3 dimensiones: Agotamiento emocional, Cinismo y Eficacia Académica. La escala de respuesta es del tipo Likert y va de 0 ("nunca") a 6 ("siempre").

- Variabilidad de la frecuencia cardíaca (HRV): Utilizando una banda transmisora y la aplicación androide Elite HVR y calculando la media RR (media de los intervalos RR), SDNN (desviación estándar de los períodos NN), y RMSSD (raíz cuadrada del valor medio de la suma de las diferencias cuadradas de todos los intervalos RR sucesivos).

\section{Procedimientos e intervención}

Después de conocer el número de estudiantes diagnosticados con agotamiento con MBI-SS, se midió la HRV usando una banda transmisora y la aplicación androide Elite HVR. Antes de las sesiones de entrenamiento, se aplicó la prueba inicial de MBI-SS y HRV. En la semana 17 se volverán a aplicar los dos instrumentos de medición a los 3 grupos y se hicieron comparaciones longitudinales y transversales (ver figura 1).

Se dividieron en tres grupos de composición similar. Un grupo de control al que no se aplicó ninguna actividad, un grupo experimental 1 al que se le hizo ejercicio aeróbico y un grupo experimental 2 al que se le aplicó un ejercicio anaeróbico (de fuerza).

En ambos grupos de intervención, el ejercicio físico se rigió por las últimas directrices para prescribir el ejercicio: Cantidad y calidad de ejercicio para desarrollar y mantener la aptitud cardiorrespiratoria, musculoesquelética y neuromotora en adultos: en la Guía para Prescribir Ejercicio, del Colegio Americano de Medicina Deportiva (ACSM) ${ }^{23}$ y se aplicaron 3 sesiones semanales durante una hora, en días alternos, durante 16 semanas.

Los ejercicios físicos fueron aplicados en las áreas de deportes de la Universidad, por un solo instructor calificado y entrenado para tal fin, con una Licenciatura en Actividad Física y Deportes.

\section{Ejercicios aeróbicos}

Se aplicaron con intensidad moderada $\mathrm{y} / \mathrm{o}$ vigorosa para la mayoría de los estudiantes. En un tiempo de 30 a 60 minutos por día (150 minutos $x$ semana) de ejercicio moderado intencional. Involucrando los principales grupos musculares y de forma continua y rítmica en su naturaleza. Incrementando el número de pasos en 2000 pasos por día para alcanzar y mantener un número de pasos diarios de 7000 pasos ${ }^{23}$.

En nuestro estudio se realizaron ejercicios aeróbicos (trotar, caminar y/o montar en bicicleta estacionaria durante aproximadamente 30 a 50 minutos, divididos en una parte inicial de calentamiento y estiramiento, una parte principal con ejercicios aeróbicos planificados y una parte final de recuperación.

\section{Ejercicios de fuerza}

Se aplicaron con una intensidad del 60\%$70 \%$ de 1 RM (intensidad moderada a dura) para principiantes a intermedios para mejorar la fuerza. Con ejercicios que involucraban a cada uno de los principales grupos musculares. Usando una variedad de equipos de ejercicio y/o peso corporal para realizarlos. De 8 a 12 repeticiones para mejorar la fuerza y la potencia. Con intervalos de descanso de 2-3 minutos entre cada serie de repeticiones. $Y$ un descanso de 48 horas entre sesiones para cada grupo muscular ${ }^{23}$.

En nuestro estudio se aplicaron ejercicios de fuerza (ejercicios de manos libres como flexiones, abdominales, abdominales, abdominales, barra fija, sentadillas de piernas, con sesiones de 30 a 50 minutos divididas en partes de calentamiento y estiramiento, la parte principal con ejercicios de fuerza planificados y la parte final de recuperación).

\section{Tratamiento estadístico de los datos}

Las estadísticas descriptivas se presentan usando la media (X) y la desviación estándar (SD). La distribución normal se probó usando la prueba de 
Shapiro-Wilk. Las comparaciones iniciales entre los grupos se hicieron usando las pruebas de KruskalWallis. Las comparaciones dentro del grupo entre los momentos previos y posteriores se hicieron usando la prueba de clasificación firmada de Wilcoxon. El cambio porcentual se calculó y presentó para cada variable. Para informar sobre la magnitud de los cambios, se calculó el tamaño del efecto de la correlación del punto biserial (rpb) y se convirtió en la d de Cohen estandarizada. Los puntos de referencia utilizados fueron: triviales $(d<0,2)$, pequeños $(d=$ $0,2$ a 0,5$)$, moderados $(d=0,5$ a 0,8$)$, grandes $(d=$ $0,8$ a 1,2$)$, muy grandes $(d>1,2)$ (Cohen, 1988). Se asumió el nivel de significación estadística para $\mathrm{p}<$ 0,05 . Se utilizó el Programa Estadístico de Ciencias Sociales (SPSS), versión 25.0 para Windows.

\section{Ética en la investigación}

La planificación de estas investigaciones se realizó teniendo en cuenta los lineamientos de la Declaración de Helsinki de 1973, revisada en 1986 y enmendada en octubre de 2013, y también se rigió por las normas estándar vigentes en la República del Ecuador para la realización de estudios biológicos. Se explicó a los estudiantes en qué consiste la investigación a fin de obtener el consentimiento informado para participar en la misma y se firmará un documento tanto por los pacientes como por el autor de la investigación para que quede constancia de ello. El estudio fue aprobado por el comité de ética correspondiente, con el código: CEISHSOLCAQ. OBS.19.129.

\section{RESULTADOS}

El diagrama de flujo de los participantes se muestra en la figura 1. El grupo de ejercicio aeróbico tuvo una edad promedio de 22,74 años $( \pm 3,05)$, En el grupo de ejercicios de fuerza fue de 22,97 años $( \pm 3,31)$, En el grupo de control, la edad promedio fue de 23,13 años $( \pm 3,77)$.

Los efectos de la intervención con ejercicios en los niveles de SB y la HRV se verificó mediante las mediciones inicial y final en los participantes de los tres grupos utilizando principalmente dos pruebas específicas para tal fin: el MBI-SS (en sus tres dimensiones; agotamiento, cinismo y eficacia académica) de corte psicológico, y la medición de la HRV (midiendo la media de los intervalos RR, la desviación estándar de los períodos NN o RR y la raíz cuadrada del valor medio de la suma de las diferencias al cuadrado de todos los intervalos RR sucesivos) de corte fisiológico. En este caso, las medias de ambas mediciones, su cambio porcentual y el cambio de tamaño del efecto se compararon utilizando el Cohen's d (véase la tabla 1).

Como puede verse en la tabla 1 , en los resultados de estas comparaciones del cambio porcentual del grupo experimental de ejercicios aeróbicos con respecto al MBI-SS en el agotamiento de la dimensión hubo un mayor porcentaje, reduciendo sus niveles en un $26,4 \%$, sobre el cinismo $(-21,06)$, y la eficacia académica $(-13,11)$. A diferencia del grupo experimental de ejercicios de fuerza en el que hubo un mayor porcentaje de cambio en la dimensión cinismo $(-27,38)$, en la eficacia $(-21,69)$, el agotamiento fue el menor cambio $(-19,55)$. En el grupo de control los niveles medios de agotamiento aumentaron desfavorablemente en un 10,26\%, también aumentó la eficacia, aunque no de forma significativa $(4,26)$, mientras que los niveles de cinismo se redujeron en un $7,26 \%$. En el grupo de intervención de ejercicios aeróbicos, la dimensión que mejoró fue el agotamiento $(d=0,532)$ con un tamaño de efecto moderado, pero no el cinismo y la eficacia que tuvieron pequeños efectos de cambio en este grupo ( $d=0,252$ y 0,397 respectivamente). En el grupo de intervención de los ejercicios de fuerza, en cambio, la dimensión que más mejoró según el tamaño del efecto fue la eficiencia, considerada moderadamente alta $(\mathrm{d}=$ 0,704 ), aquí el cambio en los niveles de agotamiento se consideró trivial y en el cinismo fue pequeño ( $d$ $=0,299$ y 0,315 respectivamente). En el grupo de control, el tamaño del efecto sobre el agotamiento, el cinismo y la eficacia se consideró trivial $(d=0,128$, 0,062 y 0,129 respectivamente) (véase la tabla 1 y los gráficos 1 y 2 ).

En cuanto a los resultados más evidentes de las pruebas de HVR, en el grupo experimental de ejercicios aeróbicos el SDNN tuvo el mayor cambio porcentual, con un aumento del $24,82 \%$, sobre la media del RR y el RMSSD $(14,40 \%$ y $16,45 \%$ respectivamente). De manera similar ocurrió en el grupo de fuerza y en el grupo de control en las tres variables anteriores con niveles de aumento sólo ligeramente inferiores en ambos grupos $(21,77 \%$, $14,24 \%, 12,60 \%$; y $12,59 \%, 4,97 \%$ y $4,99 \%$ respectivamente). 


\section{2,5}

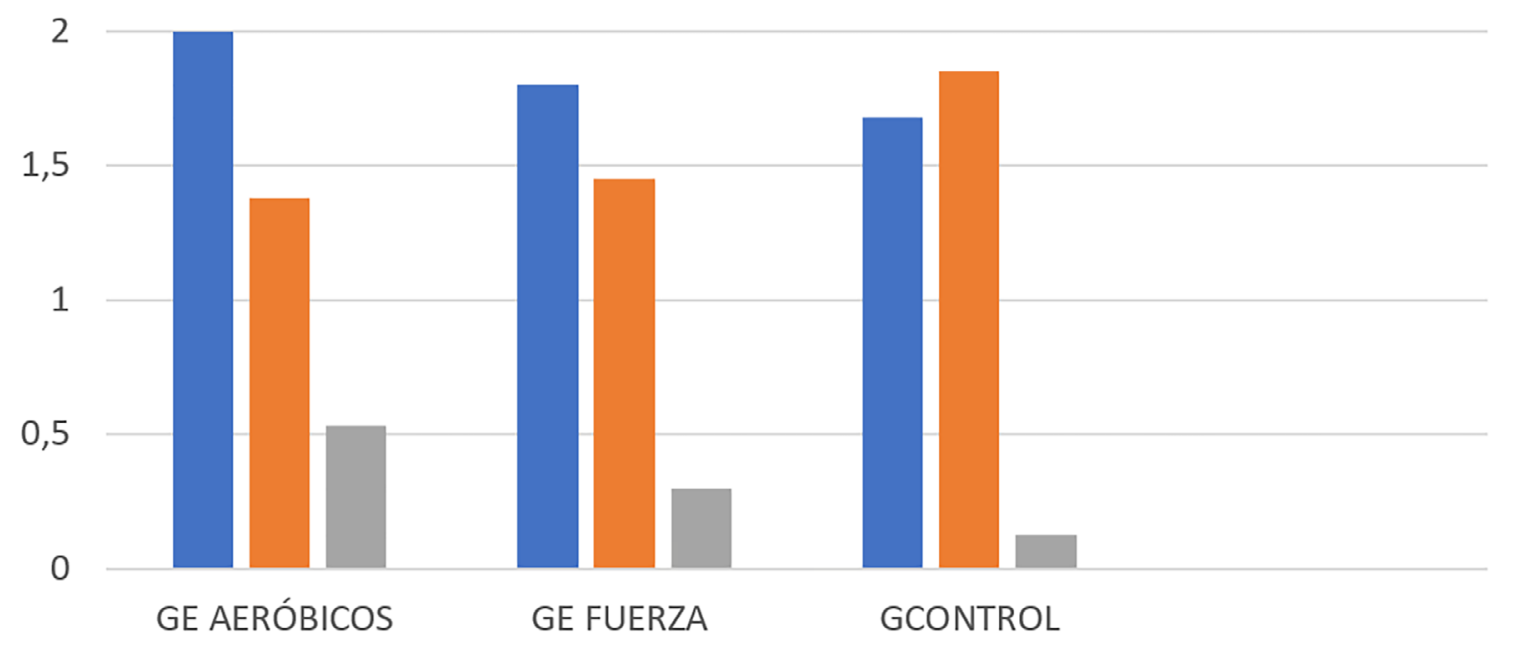

$\square$ Pretest Postest $\square$ Tamaño de efecto (d)

Gráfico 1. Comparación entre grupos según los niveles de la Dimensión: Agotamiento GE AERÓBICOS: Grupo experimental de ejercicios aeróbicos (27 participantes). GE FUERZA: Grupo experimental de ejercicios de fuerza (26 participantes). GCONTRO: Grupo control sin intervención (27 participantes).

Comparación usando la prueba de rangos de Wilcoxon para $p<.05$

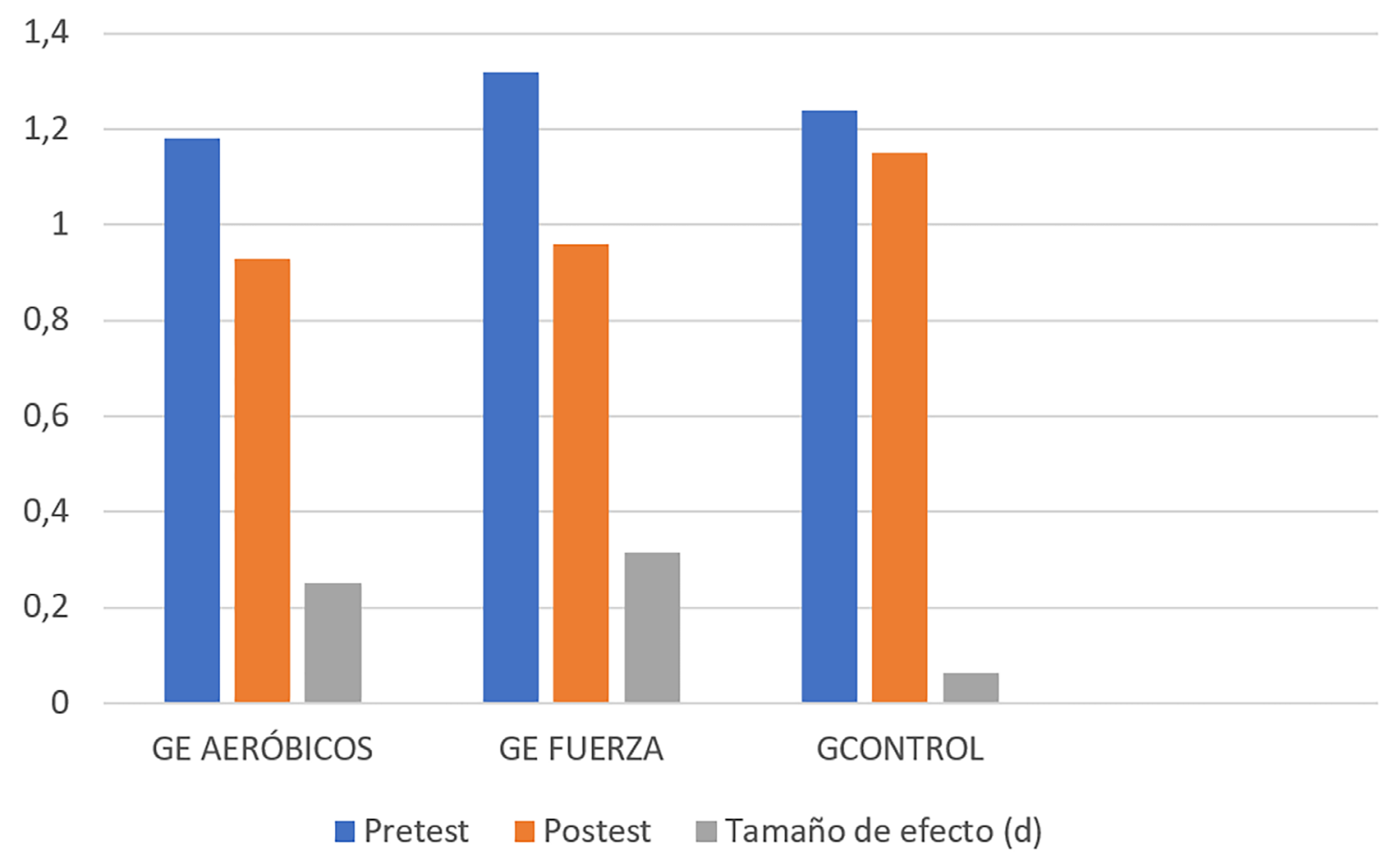

Gráfico 2. Comparación entre grupos según los niveles de la Dimensión: Cinismo Leyenda: GE AERÓBICOS: Grupo experimental de ejercicios aeróbicos. GE FUERZA: Grupo experimental de ejercicios de fuerza. GCONTRO: Grupo control sin intervención Comparación usando la prueba de rangos de Wilcoxon para $p<.05$ 
Los resultados en el tamaño del efecto mostraron un cambio muy grande en la media del RR en los grupos de aeróbicos y de fuerza $(d=1,281$ y 1,328 respectivamente), a diferencia del SDNN que fue grande y el RMSSD que fue pequeño en ambos grupos $(\mathrm{d}=0,943$ y 0,833 ; y 0,425 y 0,318 respectivamente). En cambio, en el grupo de control fueron pequeños y triviales $(0,449,0,457$ y 0,120 respectivamente).

\section{DISCUSIÓN}

El objetivo principal de este estudio fue determinar los efectos de los ejercicios físicos en los niveles de Síndrome de Burnout y la Variabilidad de la Frecuencia Cardíaca en estudiantes universitarios. Los resultados muestran que, mediante la intervención con ejercicio físico, estos

Tabla 1. Comparación entre las mediciones iniciales y finales de los grupos de intervención (ejercicios aeróbicos y de fuerza) y el grupo control (sin ejercicios).

\begin{tabular}{|c|c|c|c|c|c|c|c|c|c|c|c|c|}
\hline \multicolumn{5}{|c|}{ GE AERÓBICOS (27 participantes) } & \multicolumn{4}{|c|}{ GE FUERZA (26 participantes) } & \multicolumn{4}{|c|}{ GC (27 participantes) } \\
\hline & $\begin{array}{c}\text { PRE } \\
\text { MEDIA } \\
\text { (SD) }\end{array}$ & $\begin{array}{l}\text { POST } \\
\text { MEDIA } \\
\text { (SD) }\end{array}$ & $\begin{array}{l}\text { PORCIENTO } \\
\text { CAMBIO }\end{array}$ & $\begin{array}{l}\text { TAMAÑO } \\
\text { EFECTO } \\
\text { (d) }\end{array}$ & $\begin{array}{c}\text { PRE } \\
\text { MEDIA } \\
\text { (SD) }\end{array}$ & $\begin{array}{l}\text { POST } \\
\text { MEDIA } \\
\text { (SD) }\end{array}$ & $\begin{array}{l}\text { PORCIENTO } \\
\text { CAMBIO }\end{array}$ & $\begin{array}{l}\text { TAMAÑO } \\
\text { EFECTO } \\
\text { (d) }\end{array}$ & $\begin{array}{c}\text { PRE } \\
\text { MEDIA } \\
\text { (SD) }\end{array}$ & $\begin{array}{l}\text { POST } \\
\text { MEDIA } \\
\text { (SD) }\end{array}$ & $\begin{array}{l}\text { PORCIENTO } \\
\text { CAMBIO }\end{array}$ & $\begin{array}{l}\text { TAMAÑO } \\
\text { EFECTO } \\
\text { (d) }\end{array}$ \\
\hline $\begin{array}{l}\text { Media } \\
\text { Agotamiento }\end{array}$ & $\begin{array}{c}2.00 \pm \\
1.24\end{array}$ & $\begin{array}{c}1.38 \pm \\
.99\end{array}$ & -26.4 & .532 & $\begin{array}{c}1.80 \pm \\
1.19\end{array}$ & $\begin{array}{c}1.45 \pm \\
1.11\end{array}$ & -19.55 & .299 & $\begin{array}{c}1.68 \pm \\
1.27\end{array}$ & $\begin{array}{c}1.85 \pm \\
1.31\end{array}$ & 10.26 & .128 \\
\hline $\begin{array}{l}\text { Media } \\
\text { Cinismo }\end{array}$ & $\begin{array}{c}1.18 \pm \\
1.03\end{array}$ & $\begin{array}{c}.93 \pm \\
.97\end{array}$ & -21.06 & .252 & $\begin{array}{c}1.32 \pm \\
1.12\end{array}$ & $\begin{array}{c}.96 \pm \\
1.22\end{array}$ & -27.38 & .315 & $\begin{array}{c}1.24 \pm \\
1.22\end{array}$ & $\begin{array}{c}1.15 \pm \\
1.69\end{array}$ & -7.26 & .062 \\
\hline $\begin{array}{l}\text { Media } \\
\text { Eficacia }\end{array}$ & $\begin{array}{c}4.27 \pm \\
1.38\end{array}$ & $\begin{array}{c}3.71 \pm \\
1.49\end{array}$ & -13.11 & .397 & $\begin{array}{c}4.24 \pm \\
1.37\end{array}$ & $\begin{array}{c}3.32 \pm \\
1.29\end{array}$ & -21.69 & .704 & $\begin{array}{c}4.27 \pm \\
1.34\end{array}$ & $\begin{array}{c}4.46 \pm \\
1.65\end{array}$ & 4.26 & .129 \\
\hline $\begin{array}{l}\text { RR media } \\
(\mathrm{ms}):\end{array}$ & $\begin{array}{c}865.69 \pm \\
102.08\end{array}$ & $\begin{array}{c}1011.37 \\
\pm \\
128.32\end{array}$ & 14.40 & 1.281 & $\begin{array}{c}859.92 \\
\pm \\
100.76\end{array}$ & $\begin{array}{c}1002.77 \\
\pm 117.92\end{array}$ & 14.24 & 1.328 & $\begin{array}{c}862.53 \\
\pm \\
101.24\end{array}$ & $\begin{array}{c}907.72 \\
\pm \\
104.05\end{array}$ & 4.97 & .449 \\
\hline SDNN & $\begin{array}{c}71.32 \pm \\
20.11\end{array}$ & $\begin{array}{c}94.87 \pm \\
29.86\end{array}$ & 24.82 & .943 & $\begin{array}{c}70.34 \\
(20.18)\end{array}$ & $\begin{array}{c}89.92 \pm \\
27.24\end{array}$ & 21.77 & .833 & $\begin{array}{c}70.91 \pm \\
21.24\end{array}$ & $\begin{array}{c}81.13 \pm \\
24.27\end{array}$ & 12.59 & .457 \\
\hline RMSSD & $\begin{array}{c}68.71 \pm \\
31.10\end{array}$ & $\begin{array}{c}82.24 \pm \\
33.78\end{array}$ & 16.45 & .425 & $\begin{array}{c}69.75 \\
(31.76)\end{array}$ & $\begin{array}{c}79.81 \pm \\
32.73\end{array}$ & 12.60 & .318 & $\begin{array}{c}70.01 \pm \\
30.97\end{array}$ & $\begin{array}{c}73.69 \pm \\
31.39\end{array}$ & 4.99 & .120 \\
\hline
\end{tabular}

podrían tener un efecto de reducción de los niveles de síndrome de burnout en sus tres dimensiones, aunque, en ocasiones, esta reducción puede ser poco considerable. $\mathrm{Y}$ en el caso de a HRV los ejercicios físicos tienen un efecto contrario de incremento de sus valores, específicamente en las tres mediciones realizadas en nuestro estudio: la media de los intervalos RR, la desviación estándar de los períodos NN y la raíz cuadrada del valor medio de la suma de las diferencias cuadradas de todos los intervalos RR sucesivos. En general, este estudio se intentó proporcionar una visión más amplia de los niveles de este elemento tan importante en el conocimiento de los niveles de salud mental de esta importante población de estudiantes.

En nuestro estudio se pudo definir qué, los ejercicios físicos podrían ser eficaces, al menos según nuestros resultados, en la reducción de los niveles de SB en su enfoque tridimensional. Es decir, podría haber una mejora moderadamente pequeña en general entre los miembros de los grupos de intervención con ejercicios aeróbicos y de fuerza sobre los del grupo de control. Esto podría significar, de manera general, que había diferencias entre las pruebas iniciales y finales en los tres grupos, pero en los dos grupos de intervención con ejercicios aeróbicos y de fuerza la diferencia fue mayor que en el grupo de control en los resultados de la aplicación del MBI-SS y la HRV. Este estudio podría tener un importante significado práctico ya que, a partir de sus resultados, se podrían elaborar, proponer y validar programas de ejercicio físico más personalizados de acuerdo con los niveles generales del SB y HRV, en el caso de los estudiantes universitarios.

Sobre este último indicador coincidimos con lo expuesto por Pérez y Almirall 24 de que el esfuerzo mental, es uno de los factores estresantes de la vida académica y la HRV constituye un indicador de alta sensibilidad y especificidad de su presencia, ya que su permite evaluar objetivamente los resultados de salud proporcionados por la integración de los aspectos biológicos, psicológicos y sociales. Es un método fácil y no invasivo que permite predecir el estado general de salud del individuo. La disminución de la HRV se ha asociado a emociones negativas y a una peor salud física y psicológica. Específicamente, 
en este estudio, hay coincidencias con algunos resultados de otras situaciones similares y otras que no fueron muy coincidentes, sino todo lo contrario. Como se puede ver en el siguiente por Bonet, Parrado y Capdevila ${ }^{25}$ se indica los efectos beneficiosos del ejercicio físico en el estado de ánimo y la salud mental general en estudiantes, intervenciones con ejercicio físico, mostraron su efecto agudo en la modulación del sistema nervioso autónomo a través del análisis de la HRV. Los valores de HRV son siempre más bajos en participantes activos. Esta diferencia se debe a su entrenamiento físico; ya que están acostumbrados a hacer ejercicio físico, su metabolismo es más rápido. La influencia de la actividad física aeróbica a largo plazo y la capacidad en HRV se ha reportado repetidamente en adultos jóvenes y mayores ${ }^{26}$. La realización de ejercicio físico agudo mejora el estado de ánimo de las personas activas y no activas, aunque más significativamente en las activas 27,28 . También mejora la HRV.

Como el estudio realizado por Han et al. ${ }^{29}$, donde exploraron la efectividad de un programa de ejercicio para trabajadores de bancos y seguros con SB. En el proceso del estudio, se desarrolló un programa práctico de ejercicios en el lugar de trabajo dentro de las compañías de bancos y seguros, durante tres meses. Después del programa de ejercicios, los niveles de SB mostraron una mejora significativa en los grupos de intervención, según la comparación de la diferencia pre-postest. Entretanto, en la investigación hecha por de De Vries et al. ${ }^{30}$, se analizó el grado en que una intervención de ejercicios es efectiva para reducir los indicadores de BS en los estudiantes. Los pacientes participantes eran estudiantes con altos niveles de BS. Los participantes del grupo de intervención mostraron una mayor disminución de los niveles de BS de manera general. Estos resultados resaltan el valor del ejercicio de baja intensidad para los estudiantes universitarios con un alto nivel de BS relacionado con el estudio. Los participantes del grupo de intervención mostraron una mayor disminución de BS ( $\mathrm{t}(48)=6,82 ; \mathrm{p}=$ $<0,001$; d de Cohen $=0,90)$ que los del grupo de control ( $t(47)=3,08, p=, 003 ; d$ de Cohen $=0,46)$. Un poco más alto que nuestro caso en el que el grupo de intervención con ejercicios aeróbicos a través de la dimensión principal agotamiento el tamaño del efecto fue ligeramente inferior $(d=0,532)$, e incluso más bajo el grupo con ejercicios de fuerza. $(d=0,299)$ y el grupo de control $(d=0,128)$.
Por otro lado, Eskilsson et al. ${ }^{31}$, aplicaron un programa de entrenamiento aeróbico de 12 semanas realizado a una intensidad moderada y rigurosa para pacientes con SB. En un estudio controlado aleatorio participaron 88 pacientes diagnosticados con SB. Los pacientes fueron asignados al azar a dos grupos, un grupo de intervención con ejercicio aeróbico de 12 semanas y un grupo de control sin entrenamiento adicional. En la evaluación final, se informó de una disminución de los niveles de SB en el grupo de intervención, mientras que en el grupo de control la disminución del nivel de SB fue mucho menor. Dado que los promedios de estos resultados son más altos que los encontrados en nuestro estudio, aunque en general los tres resultados son similares en la reducción de los niveles de SB a través del ejercicio, también son muy similares a los nuestros. Otro estudio realizado por Gerber et al. ${ }^{32}$, concluyeron en su estudio que los pacientes del grupo de ejercicio entrenado aumentaron la duración de su ejercicio más que los pacientes del grupo de instrucción general. Este estudio ha demostrado que el aumento sustancial de los niveles de ejercicio puede reducir los niveles de SB en los pacientes mediante un tratamiento general integral y regular. Muy similar a nuestros resultados.

En cambio, Stenlund et al. ${ }^{33}$, evaluaron la eficacia del Qigong en la rehabilitación de pacientes con SB. Y pudieron comprobar que no había diferencias significativas en la eficacia del tratamiento entre los grupos de intervención y de control, a diferencia de nuestro estudio. Ambos grupos mejoraron significativamente con el tiempo, con una reducción de los niveles de SB y un aumento del equilibrio dinámico y la capacidad física. Finalmente, en otro estudio posterior realizado por De Vries et al..$^{34}$ se evaluó la eficacia de una intervención con ejercicios para reducir el SB en pacientes que tenían varias profesiones. Los resultados del análisis de covarianza revelaron que, el grupo de intervención informó de un nivel de SB más bajo que el grupo de control. Este estudio demuestra que el ejercicio puede considerarse una poderosa herramienta terapéutica para quienes siguen el tratamiento.

Entre las posibles fortalezas de este estudio se encuentra la correcta metodología utilizada en la investigación para determinar sus resultados cuando se realiza en tres grupos, uno de ellos de control, hechos al azar de estructura muy similar con prueba inicial y prueba final. Además, los ejercicios 
se aplicaron en los grupos de intervención siguiendo las últimas recomendaciones del ACSM ${ }^{23}$. Por otra parte, en cuanto a las limitaciones se puede afirmar que no fue posible aplicar otras pruebas relacionadas con los ejes hipotálamo-hipófisis-suprarrenal y simpático-médulo-suprarrenal, relacionadas con el sistema inmunológico y las relacionadas con la inflamación ${ }^{35}$. Para determinar la eficacia de la aplicación de los ejercicios físicos, se utilizaron en su lugar la prueba de corte psicológico y fisiológico. A pesar de que las segundas están validadas a nivel nacional e internacional y tienen una eficacia bien demostrada, habría sido interesante poder aplicar también las primeras para una mejor evaluación y solidez de los resultados.

Para futuros estudios, se podrían desarrollar, proponer y validar programas de ejercicios más específicos para la reducción de los niveles de SB en los estudiantes universitarios, tomando como base estos resultados y otros similares. Como principal conclusión, se puede exponer que, mediante la intervención con ejercicio físico, al menos en la muestra estudiada, hubo un efecto de reducción de los niveles de síndrome de burnout en sus tres dimensiones, y en el caso de a HRV los ejercicios físicos tuvieron un efecto contrario de incremento de sus valores.

\section{REFERENCIAS}

1.Segura O. Professional Exhaustion: Concepts and Implications for Public Health Biomedical. 2014; 34(4): 535-545.

2. World Health Organization. ICD-10 International Classification of Diseases. Geneva: American Psychiatric Publishing, Inc. 1994; p. 1244.

3.Freudenberger HJ. Staff burnout. J Social Issues. 1974; 30(1):159-166.

4.Cherniss C. Staff Burnout. London: Sage Publications; 1980.

5.Maslach C, Jackson SE. The Maslach Burnout Inventory. Research Edition, Palo Alto, C.A.: Consulting Psychologist Press; 1981.

6.Li H, Cheng B, Zhu XP. Quantification of burnout in emergency nurses: A systematic review and meta-analysis. Int Emerg Nurs. 2018;17. pii: S1755-599X (17)30173-8.

7.Mikalauskas A, Benetis R, Širvinskas $E$, Andrejaitienè J, Kinduris Š, Macas A, Padaiga Ž. Burnout Among Anesthetists and Intensive Care Physicians. Open Med (Wars). 2018; 5(13):105-112.

8.Armenta-Hernández OD, Maldonado-Macías A, GarcíaAlcaraz J, Avelar-Sosa L, Realyvasquez-Vargas A, Serrano-Rosa MA. Relationship between Burnout and
Body Mass Index in Senior and Middle Managers from the Mexican Manufacturing Industry. Int J Environ Res Public Health. 2018; 15(3).

9.Kavanagh KR, Spiro J. Faculty Wellness: Educator Burnout among Otolaryngology Graduate Medical Educators. Otolaryngol Head Neck Surg. 2018; 158(4):1-19.

10. Bruschini M, Carli A, Burla F. Burnout and workrelated stress in Italian rehabilitation professionals: $A$ comparison of ysiotherapists, speech therapists and occupational therapists. Work. 2018; 59(1):121-129.

11. Kawamura $Y$, Takayashiki A, Ito M, Maeno T, Seo E, Maeno T. Stress Factors Associated With Burnout Among Attending Physicians: A Cross-Sectional Study. J Clin Med Res. 2018;10(3):226-232.

12.Boni RAdS, Paiva CE, de Oliveira MA, Lucchetti G, Fregnani JHTG, Paiva BSR. Burnout among medical students during the first years of undergraduate school: Prevalence and associated factors. PLoS ONE. 2018; 13(3): e0191746

13.Erschens R, Keifenheim KE, Herrmann-Werner A, Loda T, Schwille-Kiuntke J, Bugaj TJ, Nikendei C, Huhn D, Zipfel S, Junne F. Professional burnout among medical students: Systematic literature review and metaanalysis. Med Teach. 2018; 14:1-12.

14. Liu H, Yansane AI, Zhang Y, Fu H, Hong N, Kalenderian E. Burnout and study engagement among medical students at Sun Yat-sen University, China: A cross-sectional study. Medicine (Baltimore). 2018;97(15): e0326.

15.Rammant E, Decaestecker K, Bultijnck R, Sundahl N, Ost $P$, Pauwels NS, et al. A systematic review of exercise and psychosocial rehabilitation interventions to improve health-related outcomes in patients with bladder cancer undergoing radical cystectomy. Clinical rehabilitation. 2018; 32(5): 594-606.

16. Colin PW, Liselotte ND, Patricia JE, Tait DS. Interventions to prevent and reduce physician burnout: a systematic review and meta-analysis. Lancet. 2016; 5;388(10057):2272-2281.

17. Bonet J, Parrado E, Barahona A, Capdevila L. Desarrollo y aplicación de un sistema de evaluación combinada de ejercicio físico, de alimentación y de variables psicológicas en jóvenes universitarias. Apunts. Medicina de l'Esport. 2016; 51(191): 75-83.

18. Capdevila L, Rodas G, Ocaña M, Parrado E, Pintanel M, Valero M. Variabilidad de la frecuencia cardiaca como indicador de salud en el deporte: validación con un cuestionario de calidad de vida (SF-12). Apunts Medicina de I" Esport. 2008; 43(158): 62-69.

19.González I, Gómez N, Ortiz R, Ibarra V. Ejercicio físico como tratamiento adyuvante de los trastornos mentales. Una revisión narrativa. Anales de la Facultad de Ciencias Médicas. 2018; 51(3): 27-32.

20.Azofeifa-Mora CA. Revisión de los beneficios de la intensidad y modalidades de ejercicio físico sobre el estrés psicológico. Pensar en Movimiento: Revista de ciencias del ejercicio y la salud. 2018; 16(1). 
21. Mejía IAO, Morales SC, Orellana PC, Lorenzo AF. Efecto de las actividades físicas en la disminución del estrés laboral. Revista Cubana de Medicina General Integral. 2017; 33(3):342-351.

22.Schaufeli WB, Martínez IM, Marques-Pinto A, Salanova $M$, Bakker $A B$. Burnout and engagement in university students: A cross-national study. Journal of CrossCultural Psychology. 2002; 33(5):464-481.

23.Garber CE, Blissmer B, Deschenes MR, Franklin BA, Lamonte MJ, Lee IM, et al. American College of Sports Medecine position stand. Quantity and quality of exercise to develop and maintain cardiorespiratory, musculoskeletal and neuromotor fitness in apparently healthy adults: A Guide to Prescribing Exercise. Medicine and science in sports and exercise. $2011 ; 43(7): 1334-1359$.

24.Pérez LVM, Almirall HPJ, Pérez BA. The variability of the heart frequency, an unquestionable indicator of the unit bio psychosocial. Invest Medicoquir. 2015;7(2):292-312.

25.Bonet J, Parrado E, Capdevila L. Efectos agudos del ejercicio físico sobre el estado de ánimo y la HRV / Acute Effects of Exercise on Mood And HRV. Revista Internacional de Medicina y Ciencias de la Actividad Física y el Deporte. 2017; 17(65): 85-100.

26. Albinet CT, Boucard G, Bouquet CA, Audiffren M. Processing Speed and Executive Functions in Cognitive Aging: How to Disentangle Their Mutual Relationship? Brain and Cognition. 2012; 79: 1-11.

27.Silva-Piñeiro R, Mayán-Santos JM. Beneficios psicológicos de un programa proactivo de ejercicio físico para personas mayores. Escritos de Psicología (Internet). 2016; 9(1): 24-32.

28. Leiva AP, Márquez PGO, Rodríguez CG, Navas JMM, Bozal RG. Ejercicio físico y calidad de vida en estudiantes universitarios. International Journal of Developmental and Educational Psychology. 2017; 2(1): 437-446.

29. Han HT, Ching YY, Chien TS, Chiou JC, Shu MP, Ruey YC. The Effects of Exercise Program on Burnout and Metabolic Syndrome Components in Banking and Insurance Workers. Industrial Health. 2013; 51(3):336-346.

30.De Vries JD, van Hooff MLM, Geurts SAE, Kompier MAJ. Exercise as an Intervention to Reduce Study-Related Fatigue among University Students: A Two-Arm Parallel Randomized Controlled Trial. PLoS ONE. 2016;11(3): e0152137. doi:10.1371/journal. pone.0152137

31.Eskilsson T, Slunga LJ, Malmberg HG, Stigsdotter AN and Boraxbekk $\mathrm{CJ}$. Aerobic training for improved memory in patients with stress-related exhaustion: a randomized controlled trial. BMC Psychiatry. 2017; 17:322.

32. Gerber $M$, Ingibj€org $H$, Jonsdottir EA, Lindwall $M$, Lindegard $A$. Promoting graded exercise as a part of multimodal treatment in patients diagnosed with stress-related exhaustion. Journal of Clinical Nursing. 2015; 24: 1904-1915.

33. Stenlund T, Birgander LS, Lindahl B, Nilsson L, Ahlgren C. Effects of Qigong in patients with burnout: a randomized controlled trial. Journal of rehabilitation medicine. 2009; 41(9): 761-767.

34.De Vries JD, Madelon LM, van Hooff $M L$, Sabine $A E$, Geurts SA., Michiel AJ. Exercise to reduce work-related fatigue among employees: a randomized controlled trial. Scand J Work Environ Health. 2017; 43(4): 337-349.

35.Gómez-Alcaina B, Montero-Marín MMP, Demarzo JP, García-Campayo J. Utility of biological markers in the early detection and prevention of burnout syndrome. Psychopathology. Clinical. 2013; 18 (3): 245-253 
Autor Correspondente:

Yury Rosales-Ricardo

yuryrr82@gmail.com

Editor:

Prof. Dr. Felipe Villela Gomes

Recibió: 01/07/2020

Aprobado: 01/06/2021 